OPEN ACCESS

Edited by:

Margherita Bignami, Istituto Superiore di Sanità (ISS), Italy

Reviewed by:

Przemyslaw Witek,

Warsaw Medical University, Poland

Song Li,

Army Medical University, China

Chao Tang

Nanjing General Hospital of Nanjing

Military Command, China

${ }^{*}$ Correspondence: Ming Feng

fengming@pumch.cn

${ }^{\dagger}$ These authors have contributed equally to this work

Specialty section:

This article was submitted to

Pituitary Endocrinology,

a section of the journal

Frontiers in Endocrinology

Received: 29 July 2021 Accepted: 19 October 2021 Published: 19 November 2021

Citation:

Zheng $X$, Wang $H$, Zhang W, Feng S,

Liu Y, Li S, Bao X, Lu L, Zhu H, Feng M and Wang $R$ (2021) Diagnosis,

Manifestations, Laboratory

Investigations, and Prognosis in

Pediatric and Adult Cushing's Disease

in a Large Center in China.

Front. Endocrinol. 12:749246.

doi: 10.3389/fendo.2021.749246

\section{Diagnosis, Manifestations,} Laboratory Investigations, and Prognosis in Pediatric and Adult Cushing's Disease in a Large Center in China

\author{
Xueqing Zheng ${ }^{1,2+}$, He Wang ${ }^{1,2+}$, Wentai Zhang ${ }^{1+}$, Shanshan Feng ${ }^{1}$, Yifan Liu ${ }^{1,2}$, Shuo $\mathrm{Li}^{1}$, \\ Xinjie Bao ${ }^{1}$, Lin Lu ${ }^{3}$, Huijuan Zhu ${ }^{3}$, Ming Feng ${ }^{1 *}$ and Renzhi Wang ${ }^{1}$ \\ ${ }^{1}$ Department of Neurosurgery, Pituitary Centre, Peking Union Medical College Hospital, Peking Union Medical College and \\ Chinese Academy of Medical Sciences, Beijing, China, 2 Peking Union Medical College, Chinese Academy of Medical \\ Sciences, Beijing, China, ${ }^{3}$ Department of Endocrinology, Peking Union Medical College Hospital, Peking Union Medical \\ College and Chinese Academy of Medical Sciences, Beijing, China
}

Purpose: Cushing's disease $(C D)$ is a rare disease that contributes to $70-80 \%$ hypercortisolemia, which presents similarities and differences between pediatric and adult patients, and even between male and female patients. However, the comparative study of CD between different age groups and different genders is still insufficient. The aim of the study is to make a systematic comparison to reveal the gender differences in children and adult patients of $\mathrm{CD}$, helping clinicians to provide optimal treatment for different groups of patients.

Methods: We conducted a retrospective research consisting of 30 pediatric and 392 adult CD patients in a single center in Peking Union Medical College Hospital. All 422 patients showed symptoms related to hypercortisolism and received adenoma excision surgery in the department of neurosurgery between 2014 and 2020.

Results: For the accuracy of diagnosis, the sensitivity of BIPSS at baseline in pediatric patients was lower than in adults (75 vs. 91\%, P = 0.054) but increased greatly after desmopressin stimulation (94 vs. 95\%). However, the accuracy of lateralization for BIPSS was not preferred for prediction. As for clinical manifestations, growth retardation, weight gain, hirsutism, and acne were more prevalent for children, while for adults, hypertension, osteopenia, glucometabolic disorder, easy bruising, hair loss, and weight loss were more frequently seen. As previously reported, we observed a significant difference between the male prevalence of pediatric and adult patients (50 vs. $17 \%, \mathrm{P}<0.001$ ), which was 
possibly caused by the more severe and earlier onset of a series of symptoms. Genderrelated comparison showed greater morbidity of nephrolithiasis, hypokalemia, hypertension, easy bruising, osteopenia, and striae for male patients, while irregular menses, hirsutism, and hair loss were more common for female patients. Further analysis showed that the secretory activity of the PA axis was higher for males, presenting as the more remarkable alteration of laboratory parameters and contributing to the more severe clinical manifestations. For patients treated with transsphenoidal pituitary surgery (TSS), the immediate prognosis could be predicted by operation history, invasiveness, Ki-67, and information provided by MRI, including tumor size and Knosp grading. However, we still lack methods to predict long-term prognosis.

Conclusions: Our study is the first detailed and systematic comparison between pediatric and adult CD patients. Further exploration of the impact of CD on different genders reveals a more severe and probably an earlier-onset pattern of CD for male patients.

Keywords: Cushing's disease, adrenocorticotrophin hormone, cortisol, pediatric, transsphenoidal pituitary surgery

\section{INTRODUCTION}

Cushing's disease $(\mathrm{CD})$ is a rare disease characterized by hypercortisolemia, caused by the excess secretion of adrenocorticotrophin hormone (ACTH) by a pituitary adenoma that stimulates overproduction of cortisol from the adrenal glands (1). Since hypercortisolemia can lead to a series of metabolic disorders and profoundly impact the quality of life, the accurate diagnosis and effective treatment can be very challenging yet critical. Compared with adults, CD is extremely rare in the pediatric population and results in multisystem disorder, including growth retardation, delayed sexual development, obesity, hypertension, glucose intolerance, and mood changes (2). Transsphenoidal pituitary surgery (TSS) is considered as the first-line treatment for CD, but the difficulty, effectiveness, and prognosis of TSS for children may be different from adult patients (3). However, detailed and systematic comparisons of diagnostic accuracy, clinical manifestations, laboratory investigations, and prognosis between pediatric and adult patients have seldom been performed. Previous studies have shown that different from female preponderance in adult patients, the proportion of males was much higher in pediatric patients, but the reason is still yet to know (4). Therefore, it is very meaningful to reveal the gender differences in children and adult populations on symptoms and laboratory parameters of $\mathrm{CD}$, helping clinicians to accurately diagnose and provide optimal treatment for different groups of patients.

To study the impact of $\mathrm{CD}$ on different age groups and different genders, we present a retrospective research consisting of 30 pediatric and 392 adult $C D$ patients in a single center who received pituitary adenoma excision operation by an experienced surgeon. After collecting the clinical data, we described and compared the diagnosis, clinical manifestation, laboratory investigations, and prognosis between children- and adultonset $\mathrm{CD}$, and further analyzed the gender-related differences in different age groups.

\section{MATERIALS AND METHODS}

\subsection{Patients}

The study population comprised 30 pediatric patients (age $<18$; 15 males and 15 females; mean age $13.5 \pm 3.7$ years, range $5-17$ years) and 392 adult patients (66 males and 326 females; mean age $39.6 \pm 11.5$ years, range 18-73 years) (Table 1). All 422 patients showed symptoms related to hypercortisolism and were treated in the department of neurosurgery in Peking Union Medical College Hospital (Beijing, China) between 2014 and 2020. All patients received pituitary adenoma excision operation by an experienced surgeon, MF. Among them, 65 adults and 4 children had received surgery before, 1 adult had only received radiotherapy before, and the other 326 adults and 26 children were classified as patients with the firsttime operation.

\subsection{Inclusion Criteria}

Patients were included in this study if they met the standards below:

(1) In immunohistochemical staining, excised tissue in surgery for $87 \%$ patients was confirmed as pituitary adenoma in histopathological analysis by $\mathrm{ACTH}(+)$.

(2) For $13 \%$ patients, the histopathological analysis failed to confirm the excised tissue as pituitary adenoma, which was possible due to the small size of pituitary adenoma. We consider the combination result of patients' clinical manifestations and auxiliary examinations. As mentioned above, all patients presented clinical manifestations of hypercortisolemia. Laboratory examinations include 24-h urinary free cortisol (UFC) measurement, 8 a.m. F (serum cortisol) and 8 a.m. ACTH, low-dose and high-dose dexamethasone suppression tests (LDDST and HDDST), bilateral petrosal sinus sampling (BIPSS) with desmopressin stimulation. Magnetic resonance imaging (MRI) scan also provided substantial evidence as an imaging examination. 
TABLE 1 | Basic information of pediatric and adult-onset CD.

\begin{tabular}{|c|c|c|c|}
\hline & Pediatric CD patients $(n=30)$ & Adult CD patients $(n=392)$ & $P$ value \\
\hline Gender (Female:Male) & $15: 15$ & $326: 66$ & $<0.001$ \\
\hline Age at surgery & $15.0(17.0-11.5)$ & $38.5(48.0-30.0)$ & \\
\hline Age at symptom onset & $12.0(14.3-9.0)$ & $33.0(43.0-26.0)$ & \\
\hline Course & $1.7(3.0-1.2)$ & $3.0(7.0-1.8)$ & 0.005 \\
\hline First time operation (\%) & $26(87)$ & $326(83)$ & 0.80 \\
\hline TSS surgery (\%) & $30(100)$ & $387(99)$ & 1 \\
\hline Microadenomas on MRI (\%) & $14(74)$ & $237(81)$ & 0.55 \\
\hline Invasiveness (\%) & $2(7)$ & $23(6)$ & 0.70 \\
\hline CSF leakage (\%) & $0(0)$ & $16(4)$ & 0.62 \\
\hline Immediate remission (\%) & $24(80)$ & $293(76)$ & 0.60 \\
\hline Long-term remission (\%) & $22(79)$ & $274(79)$ & 0.99 \\
\hline Recurrence (\%) & $3(11)^{a}$ & $22(6)$ & 0.42 \\
\hline
\end{tabular}

${ }^{a}$ The three recurrent pediatric $C D$ patients were all male.

The bolded values showed the significant results $(P<0.05)$. No other significant meanings were related

\subsection{Auxiliary Examinations of Cushing's Disease}

For etiologic diagnosis, an increased level of 24-h UFC indicates hypercortisolism ( $>103.5 \mu \mathrm{g} / \mathrm{d}$ ), which is further supported by increased 8 a.m. F $(>22.3 \mu \mathrm{g} / \mathrm{dl})$ and 8 a.m. ACTH $(>46 \mu \mathrm{g} / \mathrm{dl})$. Pseudo-Cushing's syndrome can be excluded by LDDST if $24-\mathrm{h}$ UFC on the second day is suppressed below $12.3 \mu \mathrm{g} / \mathrm{d}$. For localization diagnosis, suppression of 24-h UFC under $50 \%$ after HDDST test acts as strong evidence of ACTH-secreting pituitary adenoma. Chest and abdomen CT excluded patients with adrenal tumors. Preoperative pituitary MRI and BIPSS were performed for localization diagnosis. MRI with incomplete data or negative results were defined as inconclusive. MRI also provides information on the size and invasiveness (Knosp $\geq 3$ ). Due to the high cost and limited availability of CRH in China, we used BIPSS with desmopressin stimulation to indicate pituitary ACTH secretion if central to peripheral ACTH ratio $\geq 2$ preor $\geq 3$ post-stimulation. Lateralization is predicted if the interpetrosal sinus gradient $\geq 1.4$. For lateralization analysis, we excluded patients with negative or incomplete BIPSS results, presented contradictory results at different time points, and whose tumor was located on both sides.

\subsection{Surgical Treatment and Prognosis}

In total, 417 patients received first-line therapy, transsphenoidal surgery (TSS), of whom 381 patients received endoscopic TSS (eTSS) and 36 patients received microscopic TSS (mTSS); other 5 patients were treated by craniotomy, including subfrontal approach and transorbital approach in consideration of the risk of cerebrospinal fluid (CSF) leakage and scope of operation. The localization, size, shape, texture, and invasiveness of the tumor were recorded during the surgery. Microadenoma and macroadenoma were differentiated on MRI by comparing the maximum diameter with $10 \mathrm{~mm}$. Differently, microadenoma and macroadenoma measured during surgery were based on the size of the excisional part of the tumor. Invasiveness was confirmed by surgeons during the surgery if the cavernous sinus was involved by tumor tissue. All the excised tissues were sent to histopathological analysis and examined by ACTH immunohistochemical staining. Only patients who received TSS were involved in the prognostic analysis Immediate remission was defined as morning (8 a.m.) serum cortisol concentration lower than $5 \mu \mathrm{g} / \mathrm{dl}$ or 24 -h UFC lower than $20 \mu \mathrm{g} / \mathrm{d}$ in a week. Three hundred seventy-seven TSStreated patients with follow-up of more than 1 year were included in the long-term prognostic analysis.

\subsection{Statistical Analysis}

Statistical analyses were performed with SPSS Statistics 25 software (IBM Corporation, Somers, NY, USA). Continuous variables were compared by independent-samples $t$-test or Wilcoxon test, according to Shapiro-Wilk normality evaluation. Correlation for continuous variables was presented with Spearman correlation coefficient. Categorical variables were analyzed by Pearson's chi-squared test and Fisher's exact test.

\section{RESULTS}

\subsection{Diagnosis}

The effectiveness of clinical examinations of $\mathrm{CD}$ is listed in Table 2. The 24-h UFC was the most sensitive investigation (97 vs. 98\%), consistent with the previous study (5). Most patients failed to suppress 24-h UFC levels during LDDST (95 vs. $97 \%$ ) and presented positive results on HDDST (93 vs. 86\%, $\mathrm{P}=0.56$ ). The sensitivity of BIPSS test (94 vs. 96\%) was just behind 24-h UFC and LDDST, again suggesting desmopressinstimulated BIPSS as an effective alternative to CRH-stimulated BIPSS (6). There was no significant difference in the sensitivity of the above four investigations between pediatric and adult patients. In our study, we also measured the accuracy of preand post-stimulation BIPSS test separately. The sensitivity of BIPSS at baseline in pediatric patients was lower than in adults (75 vs. 91\%, $\mathrm{P}=0.054$ ) but increased after desmopressin stimulation (94 vs. 95\%). However, sampling lateralization could not predict tumor lateralization accurately for both children and adults (45 vs. 46\%). Further analysis showed that lateralization accuracy of BIPSS for macroadenomas on MRI was not higher than that for microadenomas on MRI (47 vs. $40 \%$, 
TABLE 2 | Clinical examinations of pediatric and adult-onset CD.

\begin{tabular}{|c|c|c|c|}
\hline & Pediatric CD patients $(n=30)$ & Adult CD patients $(n=392)$ & $P$ value \\
\hline 8 a.m. ACTH $>46 \mu \mathrm{g} / \mathrm{dl}$ & $23(77)$ & $301(77)$ & 0.93 \\
\hline 8 a.m. $F>22.3 \mu \mathrm{g} / \mathrm{dl}$ & $23(77)$ & $289(74)$ & 0.76 \\
\hline 24-h UFC > $103.5 \mu \mathrm{g} / \mathrm{d}$ & $28(97)$ & $370(98)$ & 0.53 \\
\hline LDDST negative & $21(95)$ & $271(97)$ & 0.50 \\
\hline HDDST positive & $26(93)$ & $291(86)$ & 0.56 \\
\hline BSIPSS sampling & $16(53)$ & $235(60)$ & 0.48 \\
\hline BSIPSS positive & $15(94)$ & $227(96)$ & 0.49 \\
\hline Pre-stimulation BSIPSS positive & $12(75)$ & $215(91)$ & 0.054 \\
\hline Post-stimulation BSIPSS positive & $15(94)$ & $224(95)$ & 0.56 \\
\hline BSIPSS lateralization concordant with surgery & $5(45)$ & $94(46)$ & 0.97 \\
\hline MRI detection & $30(100)$ & $385(98)$ & 1 \\
\hline MRI positive & $26^{a}(87)$ & $361(94)$ & 0.13 \\
\hline MRI localization concordant with surgery & $19(79)$ & $256(72)$ & 0.44 \\
\hline Microadenomas on MRI & $14(74)$ & $237(81)$ & 0.55 \\
\hline Microadenomas on MRI concordant with surgery & $12(75)$ & $245(89)$ & 0.10 \\
\hline Invasiveness & $2(7)$ & $23(6)$ & 0.70 \\
\hline MRI Knosp grading $\geq 3$ & $3(12)$ & $6(2)$ & 0.023 \\
\hline Specificity of MRI Knosp grading & $1(33)$ & $6(100)$ & 0.083 \\
\hline Sensitivity of MRI Knosp grading & $1(50)$ & $6(32)$ & 1 \\
\hline Pathology confirmed & $26(87)$ & $340(87)$ & 1 \\
\hline$K i-67 \geq 3$ & $7(25)$ & $101(27)$ & 0.79 \\
\hline
\end{tabular}

${ }^{a}$ Other four pediatric patients who were MRI negative were all male.

$F$, serum cortisol.

The bolded values showed the significant results $(P<0.05)$. No other significant meanings were related.

$P=0.59)$, raising the doubt for its effectiveness. MRI identified fewer pituitary adenomas in pediatric patients than with adult patients ( 87 vs. $94 \%, \mathrm{P}=0.13$ ). The percentage of concordance of microadenomas identified by MRI with surgery was also lower for children (75 vs. $89 \%, \mathrm{P}=0.10$ ). Compared with BIPSS, MRI was more sensitive for localization (pediatric: 79 vs. 45\%; adults: 72 vs. $46 \%$ ). However, the specificity (33 vs. 100\%, $\mathrm{P}=0.083$ ) and sensitivity (50 vs. 32\%) of Knosp grading did not achieve satisfying predictive results. After TSS, excised tissues of 27 pediatric and 365 adult patients were confirmed as ACTHsecreting pituitary adenoma.

\subsection{Manifestations}

\subsubsection{Gender Distribution}

As previously reported $(3,4,7)$, there is a significant difference between gender distribution of pediatric and adult-onset $\mathrm{CD}(\mathrm{P}<$ 0.001). Females dominated the adult CD group (83\%), while an equal gender distribution was observed among 30 pediatric patients (50 vs. 50\%). For children, male patients were older than female patients $(14.7 \pm 2.8 v s .12 .3 \pm 4.3, \mathrm{p}=0.075)$, which is different from adult patients $[36.0(48.3-28.0)$ vs. 39.0 (48.031.0), $\mathrm{p}=0.23$.

\subsubsection{Comparison of Clinical Manifestations Between Pediatric and Adult CD Patients}

Early recognition of manifestations and symptoms of $\mathrm{CD}$ is critical for timely diagnosis and treatment, especially for pediatric patients. In this study, all patients developed more than two typical clinical manifestations shown in Figure 1 and Supplementary Table 1. Similar to previous researches $(2,3,8)$, weight gain $(100 \%)$, facial changes $(93 \%)$, hirsutism $(80 \%$, as a typical sign of virilization), and growth retardation (70\%) were the most common manifestations of pediatric CD patients, among which weight gain and hirsutism were more frequently shown compared with adult patients (100 vs. 69\%, $\mathrm{P}<0.001 ; 80$ vs. $59 \%, \mathrm{P}=0.023)$. Additionally, acne was also more common in pediatric patients $(67$ vs. $47 \%, \mathrm{P}=0.040)$. For adults, hypertension were the two most common manifestations compared to pediatric patients (86 vs. 67\% $\mathrm{P}=0.007)$. Two long-term metabolic-related diseases, osteopenia and glucose intolerance or diabetes, were more common for adults (76 vs. $47 \%, \mathrm{P}<0.001 ; 64$ vs. 40\%, $\mathrm{P}=0.008)$. Besides, easy bruising and hair loss were also more frequently presented for adults (65 vs. $47 \%, \mathrm{P}=0.047 ; 18$ vs. $3 \%, \mathrm{P}=0.036$ ). Weight loss only occurred in adult patients, with $87 \%$ of whom accompanied with glucose intolerance or diabetes.

\subsubsection{Comparison of Gender-Related Manifestations Between Pediatric and Adult CD Patients}

Since the gender distribution and the pattern of clinical manifestations are different between pediatric and adult CD patients, we further analyzed the manifestations between male and female in pediatric and adult group respectively (Figures 2, 3 and Supplementary Table 2). Besides the specific presentation of irregular menses for female, two hyperandrogenemia-related manifestations, hirsutism (pediatric: 100 vs. 60\%, $\mathrm{P}=0.017$; adult: 66 vs. $25 \%, \mathrm{P}<0.001$ ) and hair loss (adult: 21 vs. $8 \%, \mathrm{P}=$ 0.013 ), are more common for females. Conversely, the prevalence of nephrolithiasis (pediatric: 47 vs. $0 \%, \mathrm{P}=0.006$; adult: 55 vs. 29\%, P < 0.001), hypokalemia (pediatric: 67 vs. 7\%, $\mathrm{P}<0.001$; adult: 61 vs. $44 \%, \mathrm{P}=0.011$ ), hypertension (pediatric: 87 vs. $47 \%, \mathrm{P}=0.020$; adult: 95 vs. $85 \%, \mathrm{P}=0.019$ ), easy bruising (pediatric: 67 vs. $27 \%, \mathrm{P}=0.028$ ), osteopenia (pediatric: 67 vs. $27 \%, \mathrm{P}=0.028$ ), and striae (adult: 65 vs. $47 \%, \mathrm{P}=0.006$ ) was 


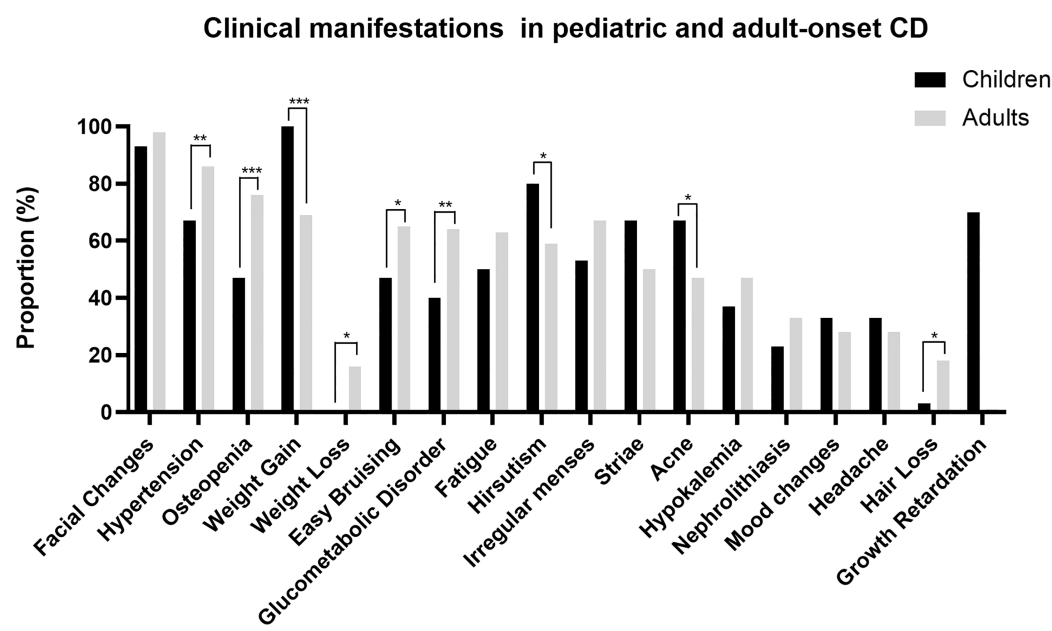

FIGURE 1 | Clinical manifestations in pediatric and adult-onset CD. ${ }^{\star} P<0.05,{ }^{\star \star} P<0.01,{ }^{\star \star \star} P<0.001$.

greater in male patients, indicating an overall more severe effects and early onset of CD on males.

\subsection{Laboratory Investigations}

\subsubsection{Comparison Between Laboratory Investigation} Results Between Pediatric and Adult CD Patients

There was no statistically significant difference in plasma ACTH and cortisol at 8 a.m. and 24-h UFC between children and adults, indicating the secretory activity of the PA axis of pediatric patients was not less than adult patients (Supplementary Table 3). For other laboratory investigations, $\mathrm{RBC}(\mathrm{P}=0.032)$ and lymphocyte count $(\mathrm{P}=0.005)$, including $\mathrm{B}$ cell $(\mathrm{p}<0.001)$ and $\mathrm{CD}^{+} \mathrm{T}$ cell count $(\mathrm{P}=0.008)$, were higher for pediatric patients. Also, a lower $\mathrm{CD} 4^{+} / \mathrm{CD}^{+} \mathrm{T}$ cell rate $(\mathrm{P}=0.050)$ and a higher level of ALT $(\mathrm{P}=0.001)$ and AST $(\mathrm{P}<0.001)$ were observed in pediatric patients.

\subsubsection{Comparison of Gender-Related Laboratory Investigation Results Between Pediatric and Adult CD Patients}

Again, we further analyzed the differences in laboratory investigation results between male and female patients in children and adults, respectively (Figure 4 and Supplementary Table 4). Serum potassium level was lower for males in both pediatric $(P=0.010)$ and adult patients $(P=0.038)$, which is consistent with the greater prevalence of hypokalemia in male patients mentioned above. For adults, the 24-h UFC $(\mathrm{P}=0.003)$ and 8 a.m. ACTH $(\mathrm{P}=0.013)$ were also higher for male patients, explaining their more severe clinical manifestations. The absolute lymphocyte count $(\mathrm{P}=0.070)$ and $\mathrm{CD} 4^{+} \mathrm{T}$ cell count $(\mathrm{P}=0.055)$ were lower for males in adult patients. Additionally, RBC $(\mathrm{P}=0.085 ; \mathrm{P}=0.085)$, ALT $(\mathrm{P}=0.048 ; \mathrm{P}=0.003)$, and testosterone level $(\mathrm{P}<0.001 ; \mathrm{P}<0.001)$ were higher for male

\section{Clinical manifestations between male and female in pediatric patients}

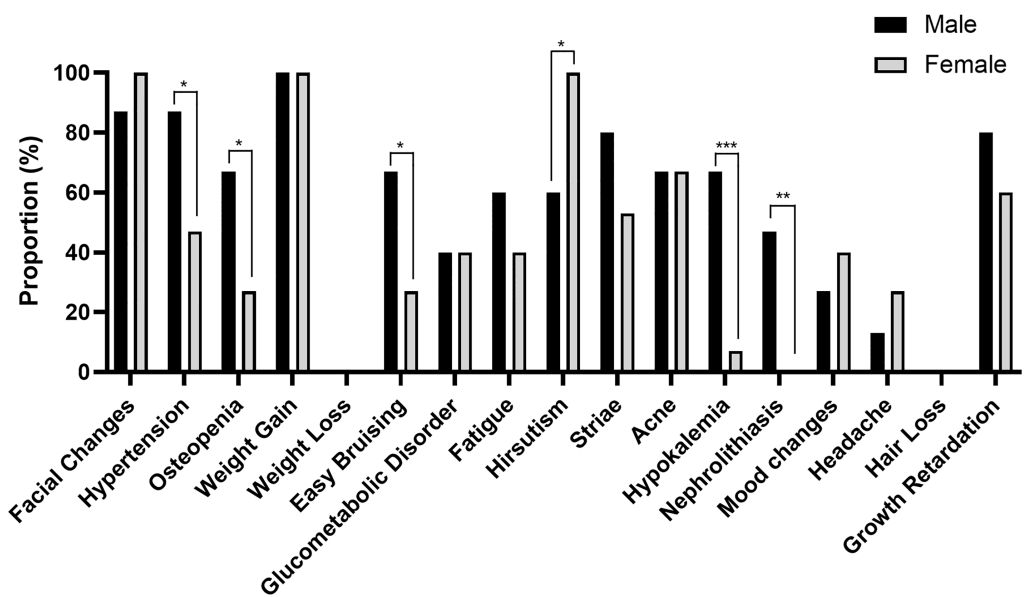

FIGURE 2 | Clinical manifestations between males and females in pediatric patients. ${ }^{\star} P<0.05,{ }^{\star \star} P<0.01,{ }^{\star \star \star} P<0.001$ 


\section{Clinical manifestations between male and female in adult patients}

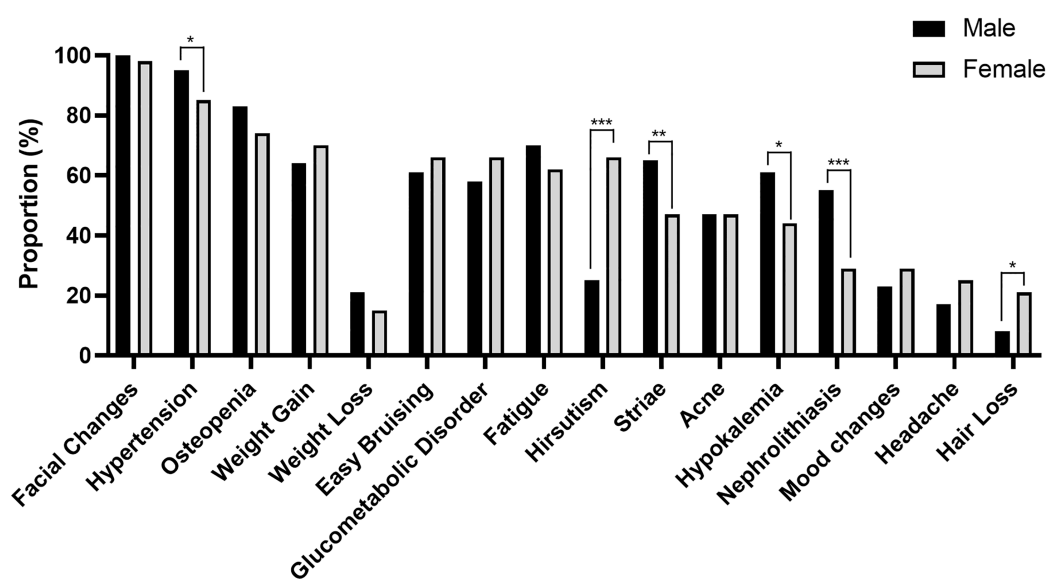

FIGURE 3 | Clinical manifestations between males and females in adult patients. ${ }^{*} P<0.05,{ }^{* \star} P<0.01,{ }^{* \star *} P<0.001$.

patients. Interestingly, body mass index (BMI) was significantly higher for males than females in pediatric patients $(P=0.012)$, while there was no such gender-related difference for adult patients.

\subsubsection{Correlations Between Pituitary-Adrenal Axis and Laboratory Investigation Results}

The 8 a.m. ACTH directly reflects the secretion level of the pituitary, controlling the release of cortisol by the adrenal gland, which is measured by 8 a.m. F level. The $24-\mathrm{h}$ UFC excretion as a sensitive investigation of hypercortisolemia is not affected by changes in cortisol-binding globulin, giving a more stable result (5). As expected, 8 a.m. ACTH level in the macroadenoma group was significantly higher at $87.3 v s .70 .9 \mu \mathrm{g} / \mathrm{dl}$ in the microadenoma group $(\mathrm{P}=0.019)$. Likewise, 8 a.m. ACTH/F was also higher for macroadenoma. However, there was no significant difference of 8 a.m. F and 24-h UFC between microadenoma and macroadenoma (Table 3). Here we use all three investigations as indicators of the PA axis and analyze the correlations with other laboratory tests for male pediatric, female pediatric, male adult, and female adult patients (Table 4).

The secretion level on the PA axis was negatively correlated with serum potassium, in concordance with the previous study (9). For male and female adult patients, 8 a.m. ACTH, 8 a.m. F, and 24-h UFC were all negatively correlated with serum potassium. While for male and female pediatric patients, only 8 a.m. ACTH or 24-h UFC was significantly correlated with serum potassium. The excess hormone production on the PA axis caused by $\mathrm{CD}$ was also correlated with decreased lymphocytes, especially $\mathrm{CD} 4^{+} \mathrm{T}$ cells. For male adult patients, the negative correlation between 8 a.m. ACTH and lymphocytes, $\mathrm{T}$ cells, and $\mathrm{CD}^{+} \mathrm{T}$ cells were observed. For female adult patients, lymphocytes, $\mathrm{T}$ cells, $\mathrm{CD} 4^{+} \mathrm{T}$ cells, $\mathrm{CD} 4^{+} \mathrm{T} / \mathrm{CD} 8^{+} \mathrm{T}$ ratio were all included. For male pediatric patients, except for lymphocytes and T cells, B cells and $\mathrm{CD}^{+} \mathrm{T}$ (with 8 a.m. ACTH: $\left.r_{s}=-0.626, P=0.053\right)$ were also correlated with the PA axis.
While for female pediatric patients, only $\mathrm{CD} 4^{+} \mathrm{T} / \mathrm{CD} 8^{+} \mathrm{T}$ ratio was correlated with 24-h UFC. Parameters of PA activity were also positively correlated with ALT for adults, and the trend of correlation of 8 a.m. ACTH and 24-h UFC with AST was also detected for male adult patients $\left(r_{s}=0.243, P=0.077 ; r_{s}=0.230\right.$, $\mathrm{P}=0.094$ ), suggesting a more severe hepatosis for adult patients. For WBC count, a positive correlation with 24-h UFC was found for male pediatric patients, and the correlation was weaker for male adults $\left(r_{s}=0.246, P=0.068\right)$. For female adult patients, we observed a negative correlation between PA activity and RBC count, and at the same time, a positive correlation between PA activity and testosterone level. Interestingly, BMI was also found to be negatively correlated with 8 a.m. F and 24-h UFC only for female adults.

\subsection{Prognosis}

We evaluated the accuracy of immediate and long-term remission prediction of the parameters of PA activity, course of the disease, size and invasiveness of tumor, Knosp grading and tumor size predicted on MRI, and whether the patient had received surgery or radiotherapy before (Table 5). For immediate prognosis, preoperative 8 a.m. ACTH was lower for the remission group $(\mathrm{P}=0.004)$. Patients who had not received surgery or radiotherapy before $(\mathrm{P}<0.001)$, with non-invasive tumor $(\mathrm{P}<0.001)$ or predicted as non-invasive by Knosp grading $(\mathrm{P}=0.008)$, with microadenoma predicted on MRI $(\mathrm{P}=0.011)$, with Ki-67 $\leq 2(\mathrm{P}=0.074)$ were more likely to achieve immediate remission. However, due to different levels of invasion, patients with different sizes of tumors resected during surgery showed no significant difference in immediate prognosis. For long-term remission and recurrence, Table 5 showed no predictor, while interestingly, preoperative 8 a.m. ACTH was higher for longterm remission group $(\mathrm{P}=0.064)$. Overall, the immediate and long-term remission rates were equal for males and females in pediatric and adult patients. The only three recurrent pediatric CD patients were all male. 

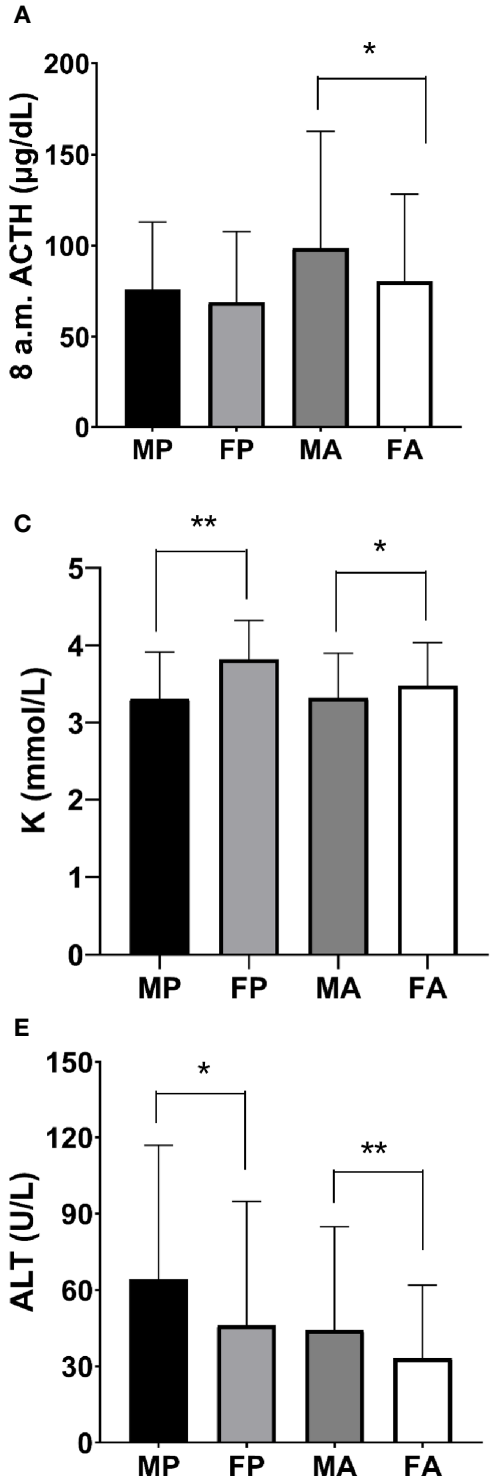

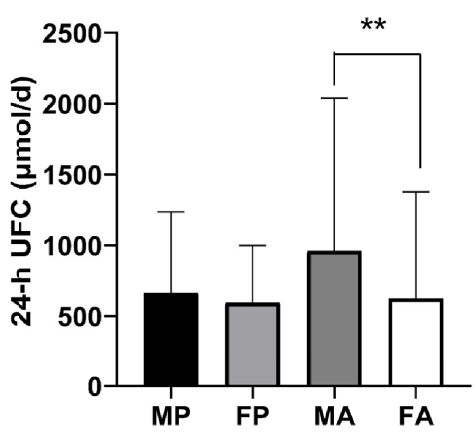

D

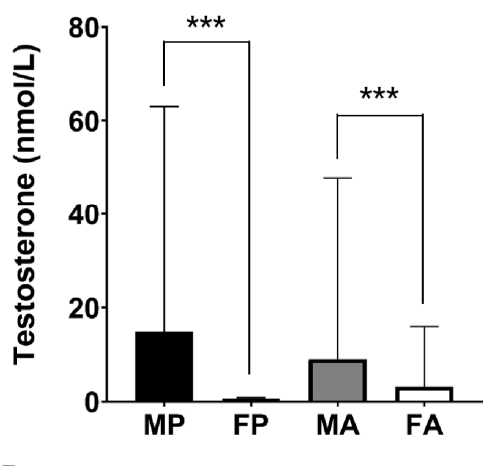

$\mathbf{F}$

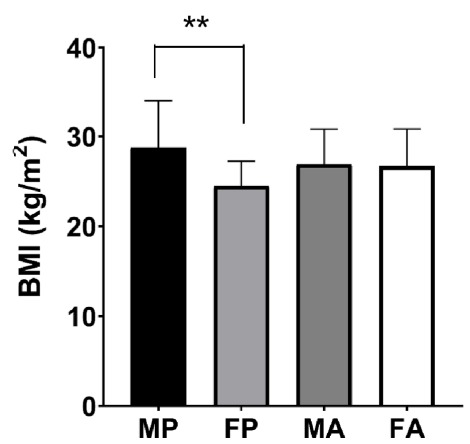

FIGURE 4 | Gender-related laboratory investigation tests in pediatric and adult-onset CD. The parameters of PA activity, (A) 8 a.m. ACTH, and (B) 24-h UFC, were significantly higher for adult male patients. The levels of (C) serum potassium, (D) testosterone, and (E) ALT were also significantly higher for males in both pediatric and adult patients. (F) BMI was significantly higher for male patients in children. MP, male pediatric patients; FP, female pediatric patients; MA, male adult patients; FA, female adult patients. $\left({ }^{\star} P<0.05,{ }^{* *} P<0.01,{ }^{* \star *} P<0.001\right)$.

\section{DISCUSSION}

As a rare disease, $\mathrm{CD}$ has a substantial impact on the quality of life of patients due to hormonal changes, especially for children in their critical growing stage. Cortisol excess will lead to growth retardation, delayed sexual development, glucose and lipid metabolism disorder, and even emotional lability (2). Thus, the differentiation and diagnosis of $\mathrm{CD}$ are very challenging and

TABLE 3 | Comparison of investigations of the PA axis between microadenoma and macroadenoma.

\begin{tabular}{|c|c|c|c|c|}
\hline & 8 a.m. ACTH ( $\mu \mathrm{g} / \mathrm{dl})$ & 8 a.m. F ( $\mu \mathrm{g} / \mathrm{dl})$ & 8 a.m. ACTH/F & 24-h UFC ( $\mu \mathrm{g} / \mathrm{d})$ \\
\hline Microadenoma on MRI & $70.9(86.1-74.3)$ & 26.9 (31.5-26.6) & $2.71(3.81-1.81)$ & 491.8 (806.4-579.3) \\
\hline Macroadenoma on MRI & $87.3(106.1-81.7)$ & $26.0(30.9-25.1)$ & $3.06(4.52-2.31)$ & $429.0(767.5-490.5)$ \\
\hline$P$ value & 0.019 & 0.78 & 0.025 & 0.61 \\
\hline
\end{tabular}

The bolded values showed the significant results $(P<0.05)$. No other significant meanings were related. 
TABLE 4 | Correlation $\left(r_{s}\right)$ between indicators of the PA axis and other laboratory investigation results.

\begin{tabular}{|c|c|c|c|c|c|c|c|c|c|c|c|c|c|c|}
\hline Patient classification & $\begin{array}{l}\text { Indicators of PA } \\
\text { axis }\end{array}$ & WBC & RBC & ALT & AST & $\mathbf{K}$ & Lymphocyte & B cell & $\mathrm{T}$ cell & $\mathrm{CD}^{+}{ }^{+} \mathrm{T}$ & $\begin{array}{c}\mathrm{CD}^{+} \\
\mathrm{T}\end{array}$ & $\begin{array}{c}\mathrm{CD4}^{+} \mathrm{T} / \mathrm{CD}^{+} \mathrm{T} \\
\text { ratio }\end{array}$ & BMI & Testosterone \\
\hline \multirow{3}{*}{$\begin{array}{l}\text { Male pediatric } \\
\text { patients }\end{array}$} & 8 a.m. ACTH & 0.352 & -0.158 & 0.091 & -0.143 & $-0.586^{\star}$ & $-0.790^{\star \star}$ & $-0.663^{*}$ & -0.547 & -0.626 & -0.182 & -0.438 & 0.070 & 0.344 \\
\hline & 8 a.m. F & 0.121 & 0.174 & 0.022 & 0.002 & -0.291 & -0.600 & 0.079 & $-0.697^{\star}$ & -0.467 & -0.467 & -0.212 & 0.000 & -0.014 \\
\hline & 24-h UFC & $0.538^{\star}$ & 0.297 & -0.059 & -0.229 & -0.138 & -0.527 & -0.297 & -0.345 & -0.285 & -0.455 & 0.006 & 0.293 & -0.215 \\
\hline \multirow{3}{*}{$\begin{array}{l}\text { Female pediatric } \\
\text { patients }\end{array}$} & 8 a.m. ACTH & 0.077 & 0.088 & 0.548 & -0.186 & 0.033 & -0.500 & 0.143 & -0.500 & -0.238 & -0.333 & -0.048 & -0.118 & 0.564 \\
\hline & 8 a.m. F & 0.396 & 0.148 & -0.096 & 0.095 & -0.215 & 0.643 & 0.143 & 0.595 & 0.143 & 0.571 & -0.238 & 0.129 & 0.064 \\
\hline & 24-h UFC & 0.105 & 0.245 & 0.375 & -0.105 & $-0.632^{\star}$ & -0.179 & -0.200 & -0.107 & -0.643 & 0.143 & $-0.786^{\star}$ & 0.002 & 0.164 \\
\hline \multirow[t]{3}{*}{ Male adult patients } & 8 a.m. ACTH & 0.008 & -0.170 & $0.457^{\star \star}$ & 0.243 & $-0.407^{\star \star}$ & $-0.367^{\star}$ & 0.028 & $-0.373^{\star}$ & $-0.392^{\star}$ & -0.266 & -0.218 & 0.041 & -0.222 \\
\hline & 8 a.m. F & 0.246 & -0.139 & $0.449^{\star \star}$ & 0.213 & $-0.380^{\star \star}$ & -0.023 & 0.282 & -0.031 & -0.099 & -0.081 & -0.245 & 0.035 & -0.211 \\
\hline & 24-h UFC & 0.014 & -0.206 & $0.437^{\star \star}$ & 0.230 & $-0.456^{\star \star}$ & -0.073 & 0.320 & -0.117 & -0.119 & -0.020 & -0.219 & -0.144 & -0.233 \\
\hline \multirow{3}{*}{$\begin{array}{l}\text { Female adult } \\
\text { patients }\end{array}$} & 8 a.m. ACTH & -0.083 & $-0.180^{\star \star}$ & 0.093 & 0.080 & $-0.145^{\star \star}$ & -0.155 & 0.031 & -0.153 & -0.211 & -0.060 & $-0.182^{*}$ & -0.094 & 0.033 \\
\hline & 8 a.m. F & 0.013 & $-0.174^{\star \star}$ & $0.136^{\star}$ & 0.051 & $-0.244^{\star \star}$ & $-0.214^{\star \star}$ & -0.017 & $-0.235^{\star \star}$ & $-0.321^{\star \star}$ & -0.148 & $-0.160^{*}$ & $-0.123^{\star}$ & $0.118^{\star}$ \\
\hline & 24-h UFC & 0.017 & $-0.208^{\star \star}$ & $0.126^{*}$ & -0.028 & $-0.189^{\star \star}$ & -0.152 & 0.027 & -0.134 & $-0.203^{\star}$ & -0.005 & $-0.204^{*}$ & $-0.197^{\star \star}$ & 0.016 \\
\hline
\end{tabular}

${ }^{*}$ Correlation is significant at the 0.05 level.

${ }^{* *}$ Correlation is significant at the 0.01 level.

essential. Several studies paid attention to pediatric CD patients, and Storr focused on comparing diagnostic and clinical features between pediatric and adult-onset CD. To our knowledge, there is no reported systematic comparison on the accuracy of diagnosis, clinical features, laboratory investigations, and prognosis between pediatric and adult $\mathrm{CD}$, and at the same time, considering the impact of $\mathrm{CD}$ on different gender.

\subsection{Accuracy and Clinical Value of Different Diagnostic Methods}

In our cohort, 24-h UFC was the most accurate investigation for hypercortisolemia for both pediatric and adult patients. BIPSS with desmopressin stimulation yielded comparable accuracy to 24-h UFC measurement. Though the sampling rate was relatively low (Table 2) as an invasive examination, BIPSS could confirm $100 \%$ 24-h UFC < $103.5 \mu \mathrm{g} / \mathrm{d}, 96 \%$ MRI negative, 97\% HDDST negative cases as $\mathrm{CD}$. It is beneficial as an accessory examination, especially for its specificity and high accuracy in distinguishing
CD and EAS $(6,10)$. We found that the sensitivity of BIPSS at baseline was lower than after stimulation, especially for children, indicating that the stimulation of desmopressin is very necessary to increase the sensitivity of BIPSS in these cases, verifying that desmopressin is an efficient alternative to CRH (6). Similarly, such an increase was also observed in the previous study (10). Chen reported that the optimal cutoff of BIPSS was 1.4 before stimulation and 2.8 after stimulation based on a cohort including $226 \mathrm{CD}$ and 24 ectopic adrenocorticotropin syndrome (EAS) patients in the endocrinology department also in Peking Union Medical College Hospital (11). Using the new criteria, the sensitivity of baseline BIPSS for children in our cohort reached $94 \%$. The specificity and accuracy for differentiation diagnosis of this new criteria still need to be confirmed for pediatric patients. For lateralization, as several studies indicated, the concordance with surgery was disappointing $(10,12,13)$. Since the asymmetric parasellar venous drainage pattern and asymmetric bilateral catheter positioning could all diminish the predictive

TABLE 5 | Prognosis of patients treated with TSS.

\begin{tabular}{|c|c|c|c|}
\hline & Immediate remission (\%) & Long-term remission (\%) & Recurrence (\%) \\
\hline Excisional microadenoma & $280(77)$ & $264(79)$ & $20(6)$ \\
\hline Excisional macroadenoma & $25(66)$ & $21(68)$ & $3(10)$ \\
\hline$P$ value & 0.14 & 0.14 & 0.43 \\
\hline Microadenoma on MRI & $203(81)$ & $45(80)$ & $4(7)$ \\
\hline Macroadenoma on MRI & $39(66)$ & $182(78)$ & $16(7)$ \\
\hline$P$ value & 0.011 & 0.75 & 1 \\
\hline Invasive & $8(0.35)$ & $15(68)$ & $1(5)$ \\
\hline Non-invasive & $310(79)$ & $273(79)$ & $24(7)$ \\
\hline$P$ value & $<0.001$ & 0.28 & 1 \\
\hline Knosp $\geq 3$ & $3(33)$ & $6(75)$ & $0(0)$ \\
\hline Knosp $\leq 2$ & $259(77)$ & $243(80)$ & $17(6)$ \\
\hline$P$ value & 0.008 & 0.67 & 1 \\
\hline First-time operation & $283(79)$ & $247(79)$ & $21(7)$ \\
\hline Non-first-time operation & $34(50)$ & $49(78)$ & $4(6)$ \\
\hline$P$ value & $<0.001$ & 0.88 & 1 \\
\hline $\mathrm{Ki}-67 \geq 3$ & $76(70)$ & $74(77)$ & $5(5)$ \\
\hline $\mathrm{Ki}-67 \leq 2$ & 228 (79) & $203(78)$ & $19(7)$ \\
\hline$P$ value & 0.074 & 0.84 & 0.48 \\
\hline
\end{tabular}

The bolded values showed the significant results $(P<0.05)$. No other significant meanings were related. 
value, we do not recommend using BIPSS to predict lateralization of pituitary adenoma (14).

Localization on MRI was more precise, yet the predictive value of tumor invasiveness on MRI still needed improvement. Also, some microadenomas could not be exactly seen on MRI scan. Similar to Storr's research, a lower percentage of adenomas were confirmed on MRI in children, and the concordance of tumor size on MRI with surgery was also lower for them. However, different from previous discoveries, there was no difference in the percentage of microadenoma on MRI between children and adults (3). As for the relationship between tumor size and CD, some previous studies with smaller sample sizes reached different conclusions. Mathioudakis and Kakade found that macroadenomas had lower serum cortisol in comparison with microadenomas $(9,15)$, while Witek discovered higher ACTH secretion by macroadenomas (16). But a higher ACTH/ serum cortisol ratio for macroadenomas seemed to be the same result. Similar to Witek, in our study, only 8 a.m. ACTH level and 8 a.m. ACTH/F were higher in the macroadenoma group, suggesting that macroadenoma was not necessarily correlated with a higher response in the lower PA axis, and thus the severity of CD.

Except for the preoperative ACTH level, invasiveness, and Ki67, we mainly depend on MRI to provide clues, including the size and Knosp grading (16), to predict immediate prognosis, illustrating that MRI indeed contains a lot of information. But still, the long-term prognosis is hard to predict by existing clinical examinations, calling for the use of radiomics. Besides, the development of machine learning also helped to optimize the algorithm of immediate and delayed remission prediction, as well as automatically identify $\mathrm{CD}$ patients by recognizing anomalies in facial images $(17,18)$.

\subsection{CD's Impact on Laboratory Parameters and Clinical Manifestations for Pediatric and Adult Patients}

For laboratory investigations, we observed a higher level of RBC count, lymphocyte count including B cell and CD8 ${ }^{+} \mathrm{T}$ cell, ALT, and AST for pediatric patients. ALT and AST are not only distributed in the liver but also stored in the skeletal muscle, indicating the level of growth and development $(19,20)$. So it was not surprising to see a higher range of ALT and AST for children. Only for adult patients did we observe a positive correlation of the secretion along the PA axis with ALT and AST, implying the relation of $\mathrm{CD}$ and liver dysfunction for adults. Blood cell counts vary with age. The absolute numbers of $\mathrm{T}$ and $\mathrm{B}$ cells for children are higher and gradually decrease to adult levels at about 13 years old (21), while RBC count gradually increases with age to a maintenance level (22). So it seems that the higher RBC count and lower $\mathrm{CD}^{+} / \mathrm{CD}^{+} \mathrm{T}$ cell for pediatric patients could not be partially explained by physiological changes. In addition, since the absence of the control group of healthy children, these parameters still need to be carefully interpreted. For other investigations, including the activity of the PA axis, because there was no significant difference between children and adult groups, the impact of $\mathrm{CD}$ on growing children might be more profound and lasting, calling for special attention. Comparing the clinical features, children tended to have a more rapid onset and obvious manifestations, including weight gain and growth retardation, driving them to seek treatments. Similar to Storr's research, we found that children were more sensitive to the change of sex hormone, particularly androgen, presented as a higher prevalence of hirsutism and acne. The suppressive effect of hypercortisolemia on the pituitary-gonadal (PG) axis have been widely reported (23). At the same time, excess circulating ACTH will stimulate androgen production in the adrenal cortex (24). Since the development of children's gonads is not mature enough, the suppressed gonadal androgen can be compensated by the excess androgen, contributing to the virilization of pediatric CD patients. But the suppression of PG function on children's immature gonads can be more alarming because of its impact on delayed sexual development (2). In Supplementary Table 1, we included all female patients. For patients between menophania and menopause, all pediatric patients developed irregular menses, while the ratio for adults was $78 \%$, indicating a strong impact on children. Furthermore, hypertension, osteopenia, glucose intolerance, fatigue, bruising, striae, mood change, and hypokalemia were also prevalent for pediatric patients and would probably further progress and develop into lifelong complications. Therefore, accurate diagnosis and early treatment are crucial to pediatric patients' normalization of metabolism and development both physically and mentally (7, 25, 26).

For adult patients, the prevalence of chronic diseases and metabolic syndromes was higher, including hypertension, osteoporosis, muscle weakness, fatigue, impaired glucose metabolism that explains weight loss, hypokalemia, nephrolithiasis, and androgen-related alopecia. Some of the manifestations, particularly hypertension and abnormal glucose metabolism, need special attention because they are related to a higher risk of cardiovascular and cerebrovascular diseases, which are the most common causes of death (27). Note that age is also an important influence factor for many of the chronic diseases mentioned above, so the long-term management and the differentiation of CD with these kinds of diseases are critical.

\subsection{CD's Impact on Laboratory Parameters and Clinical Manifestations for Male and Female Patients}

Gender distribution in pediatric CD patients was analyzed in many studies, some of which reported male predominance. Same as a large series of 102 patients from the NIH, our study showed an equal gender distribution (7). So far, the reason for the higher male proportion in pediatric patients compared with adults is not clear. By comparing the manifestations, laboratory investigations, and prognosis between different gender in the pediatric and adult groups, we speculated that male patients develop more severe symptoms at a younger age. Comparing the PA activity, we found that 8 a.m. ACTH and 24-h UFC were significantly higher for males in the adult group despite the prevalence of microadenomas on MRI was similar for different gender, indicating a more pronounced secretory activity. 
Whereas for children, male patients did not present higher PA activity, but excess cortisol seemed to have a more profound influence on their growing bodies under different levels of sex hormones, resulting in more severe clinical manifestations.

Correlation analysis showed that the level of serum potassium and the number of lymphocytes, especially $\mathrm{CD} 4^{+} \mathrm{T}$ cells, were negatively correlated with parameters of PA secretion level for both genders in children and adults. Cortisol can bind to the mineralocorticoid receptor on the collecting tubule and promote the excretion of potassium and reabsorption of sodium, contributing to hypokalemia. Also, a previous study has reported the negative correlation between plasma potassium and cortisol levels (28). As for the immune regulation of CD, lymphopenia, reduced $\mathrm{CD} 4^{+} \mathrm{T}$ cell count, and a reduced ratio of $\mathrm{CD}^{+} / \mathrm{CD}^{+} \mathrm{T}$ cells have been reported in $\mathrm{CD}$ patients (8). In contrast, only the absolute lymphocyte count has been demonstrated to be negatively correlated with serum cortisol and 24-h UFC level $(29,30)$. The mechanism of lymphopenia has been partially revealed by an in vitro study that $\mathrm{T}$ cells were sensitive to GC-induced apoptosis, especially for $\mathrm{CD}^{+} \mathrm{T}$ cells (31). However, this laboratory finding could not explain the reduced ratio of $\mathrm{CD} 4^{+} / \mathrm{CD}^{+} \mathrm{T}$ cells and other cell types linked to $\mathrm{CD}$.

In the present study, we found that hypokalemia was more prevalent for males in both pediatric and adult patients, and the absolute serum potassium level was also lower for males. The significant difference in the degree of hypokalemia between different genders has not been reported and explained. For the immune system, we found that lymphocytes and $\mathrm{CD} 4^{+} \mathrm{T}$ cells were lower for adult male patients, while the number of lymphocytes, B cells, $\mathrm{T}$ cells, and $\mathrm{CD} 4^{+} \mathrm{T}$ cells also seemed to be lower for pediatric male patients. The overall more profound effect of immune suppression for male patients implies the higher possibility of infections, which is related to a higher risk of mortality $(8,29)$. Another gender-related parameter was BMI, which was significantly higher for males in pediatric patients, possibly resulting in the early care-seeking behavior for male children. The increased adipose tissue will lead to metabolic changes, including leptin, resistin, and pro-inflammatory factors, leading to insulin resistance and other cardiovascular and cerebrovascular diseases (8). Considering other clinical manifestations, we found that female-prevalent symptoms were all complications of hyperandrogenism, consisting of alopecia and hirsutism. That's because, compared with male adults, the PG function only accounts for half of the total androgen. Therefore, the excess ACTH secretion could have an opposite impact on serum testosterone levels for male and female CD patients. For other manifestations, the prevalence of nephrolithiasis and hypertension was more remarkable for male patients. Osteoporosis and ecchymosis were more common for males in the pediatric group, indicating the early onset of these symptoms for males. Furthermore, striae presence was also higher for males in adult patients.

In summary, the secretory activity of the PA axis was higher for males, contributing to the more remarkable alteration of long-term metabolism and immune system, presenting as more severe and earlier onset of a series of symptoms. Besides, the pituitary adenoma was less confirmed by MRI and recurred more frequently after TSS surgery for males in the pediatric group, supporting the more dangerous nature of pituitary adenoma for male patients. But the mechanism behind the gender-related difference in the characteristics of CD is still unknown. The results of our study were similar to previous literature, which concluded that male CD patients showed more severe clinical presentation, higher ACTH and cortisol levels (32). However, our study recruited more patients and compared the genderrelated differences in the pediatric and adult group, achieving more significant results supporting the more serious CD in males.

\subsection{Limitations and Strengths}

Our study has several limitations. First, we did not recruit a control group of matched healthy children and adults, so we could not get a clearer view of the degree of CD's impact on different kinds of patients. Second, we did not include patients showing hypercortisolemia caused by other diseases such as EAS. Therefore, we could not measure the specificity of some diagnostic methods. Third, we screened patients with followup visits longer than 1 year for the long-term prognosis part. However, the time was still not long enough to observe the recovery of chronic and metabolic complications of CD. Fourth, because this is a retrospective study, we could not avoid missing values, especially for the results of laboratory investigations that were recorded postoperatively and during follow-up visits. Without the comparison between pre- and post-operation laboratory tests, we could not exclude the heterogeneity between different patients and evaluate the change after TSS surgery. Last, although we included a large number of 422 patients in total, the number of pediatric patients was relatively insufficient due to lower morbidity, limiting the accuracy and significance of the results related to children.

Our study contains the largest single-center cohort including both pediatric and adult $\mathrm{CD}$ patients, contributing high-value and reliable clinical evidence to the preclinical and clinical field of CD-related researches. Compared with other studies that compared the impact of CD on different groups of patients, we are the first to consider both factors of age and gender. Additionally, all patients received pituitary adenoma excision by only one experienced surgeon, controlling the impact of operational heterogeneity on prognosis. We successfully observed a more severe and probably an earlier-onset pattern of $\mathrm{CD}$ for male patients, reminding the clinicians during their clinical practices, and at the same time, promoting deeper researches into the mechanism of pathogenesis of $\mathrm{CD}$ for different patient groups.

To conclude, in this paper, we present a retrospective study comparing the diagnosis, symptoms, preoperative laboratory parameters, and prognosis of $\mathrm{CD}$ for pediatric and adult patients. To explore the reason for the higher male proportion in pediatric $C D$ patients, we further analyzed the impact of $C D$ on different genders in children and adults, observing a more severe and probably an earlier-onset pattern of $\mathrm{CD}$ for male patients. 


\section{DATA AVAILABILITY STATEMENT}

Dataset is accessible through contact with the corresponding author. Requests to access these datasets should be directed to XZ, 1608596426@qq.com.

\section{ETHICS STATEMENT}

The studies involving human participants were reviewed and approved by the Institutional Review Board of Peking Union Medical College Hospital. Written informed consent from the participants' legal guardian/next of kin was not required to participate in this study in accordance with the national legislation and the institutional requirements.

\section{AUTHOR CONTRIBUTIONS}

XZ: Conceptualization, methodology, formal analysis, investigation, data curation, writing-original draft, visualization. HW: Methodology, writing-original draft.

\section{REFERENCES}

1. Sharma ST, Nieman LK, Feelders RA. Comorbidities in Cushing's Disease. Pituitary (2015) 18(2):188-94. doi: 10.1007/s11102-015-0645-6

2. Keil MF. Quality of Life and Other Outcomes in Children Treated for Cushing Syndrome. J Clin Endocrinol Metab (2013) 98(7):2667-78. doi: 10.1210/jc.2013-1123

3. Storr HL, Alexandraki KI, Martin L, Isidori AM, Kaltsas GA, Monson JP, et al. Comparisons in the Epidemiology, Diagnostic Features and Cure Rate by Transsphenoidal Surgery Between Paediatric and Adult-Onset Cushing's Disease. Eur J Endocrinol (2011) 164(5):667-74. doi: 10.1530/eje-10-1120

4. Libuit LG, Karageorgiadis AS, Sinaii N, Nguyen May NM, Keil MF, Lodish MB, et al. A Gender-Dependent Analysis of Cushing's Disease in Childhood: Pre- and Postoperative Follow-Up. Clin Endocrinol (Oxf) (2015) 83(1):72-7. doi: $10.1111 /$ cen.12644

5. Shapiro L, Elahi S, Riddoch F, Perry LA, Martin L, Akker SA, et al. Investigation for Paediatric Cushing's Syndrome Using Twenty-Four-Hour Urinary Free Cortisol Determination. Horm Res Paediatr (2016) 86(1):21-6. doi: 10.1159/000446913

6. Feng M, Liu Z, Liu X, Zhang X, Bao X, Yao Y, et al. Tumour Lateralization in Cushing's Disease by Inferior Petrosal Sinus Sampling With Desmopressin. Clin Endocrinol (Oxf) (2018) 88(2):251-7. doi: 10.1111/cen.13505

7. Ferrigno R, Hasenmajer V, Caiulo S, Minnetti M, Mazzotta P, Storr HL, et al. Paediatric Cushing's Disease: Epidemiology, Pathogenesis, Clinical Management and Outcome. Rev Endocr Metab Disord (2021). doi: 10.1007/ s11154-021-09626-4

8. Ntali G, Grossman A, Karavitaki N. Clinical and Biochemical Manifestations of Cushing's. Pituitary (2015) 18(2):181-7. doi: 10.1007/s11102-014-0631-4

9. Mathioudakis N, Pendleton C, Quinones-Hinojosa A, Wand GS, Salvatori R. ACTH-Secreting Pituitary Adenomas: Size Does Not Correlate With Hormonal Activity. Pituitary (2012) 15(4):526-32. doi: 10.1007/s11102-011-0362-8

10. Chen S, Chen K, Lu L, Zhang X, Tong A, Pan H, et al. The Effects of Sampling Lateralization on Bilateral Inferior Petrosal Sinus Sampling and Desmopressin Stimulation Test for Pediatric Cushing's Disease. Endocrine (2019) 63(3):58291. doi: 10.1007/s12020-018-1779-x

11. Chen S, Chen K, Wang S, Zhu H, Lu L, Zhang X, et al. The Optimal Cut-Off of BIPSS in Differential Diagnosis of ACTH-Dependent Cushing's Syndrome: Is Stimulation Necessary? J Clin Endocrinol Metab (2020) 105(4):e1673-85. doi: 10.1210/clinem/dgz194

12. McNally PG, Bolia A, Absalom SR, Falconer-Smith J, Howlett TA. Preliminary Observations Using Endocrine Markers of Pituitary Venous
WZ: Conceptualization, methodology, data curation. SF: Methodology, data curation, resources. YL: Investigation. SL: Investigation. XB: Data curation, resources. LL: Data curation, resources. HZ: Data curation, resources. MF: Conceptualization, methodology, validation, writing-review and editing, supervision, project administration. RW: Supervision, project administration. All authors contributed to the article and approved the submitted version.

\section{FUNDING}

This work was supported by the reforming programme on education for graduates in PUMC (10023201900107).

\section{SUPPLEMENTARY MATERIAL}

The Supplementary Material for this article can be found online at: https://www.frontiersin.org/articles/10.3389/fendo.2021.749246/ full\#supplementary-material

Dilution During Bilateral Simultaneous Inferior Petrosal Sinus Catheterization in Cushing's Syndrome: Is Combined CRF and TRH Stimulation of Value? Clin Endocrinol (Oxf) (1993) 39(6):681-6. doi: 10. 1111/j.1365-2265.1993.tb02427.x

13. Kaskarelis IS, Tsatalou EG, Benakis SV, Malagari K, Komninos I, Vassiliadi D, et al. Bilateral Inferior Petrosal Sinuses Sampling in the Routine Investigation of Cushing's Syndrome: A Comparison With MRI. AJR Am J Roentgenol (2006) 187(2):562-70. doi: 10.2214/ajr.05.0557

14. Ghorbani M, Akbari H, Griessenauer CJ, Wipplinger C, Dastmalchi A, Malek M, et al. Lateralization of Inferior Petrosal Sinus Sampling in Cushing's Disease Correlates With Cavernous Sinus Venous Drainage Patterns, But Not Tumor Lateralization. Heliyon (2020) 6(10):e05299. doi: 10.1016/ j.heliyon.2020.e05299

15. Kakade HR, Kasaliwal R, Khadilkar KS, Jadhav S, Bukan A, Khare S, et al. Clinical, Biochemical and Imaging Characteristics of Cushing's Macroadenomas and Their Long-Term Treatment Outcome. Clin Endocrinol (Oxf) (2014) 81(3):336-42. doi: 10.1111/cen.12442

16. Witek P, Zieliński G, Szamotulska K, Maksymowicz M, Kamiński G. Clinicopathological Predictive Factors in the Early Remission of Corticotroph Pituitary Macroadenomas in a Tertiary Referral Centre. Eur J Endocrinol (2016) 174(4):539-49. doi: 10.1530/eje-15-1226

17. Fan Y, Li Y, Bao X, Zhu H, Lu L, Yao Y, et al. Development of Machine Learning Models for Predicting Postoperative Delayed Remission in Patients With Cushing's Disease. J Clin Endocrinol Metab (2021) 106(1):e217-e31. doi: $10.1210 /$ clinem/dgaa698

18. Wei R, Jiang C, Gao J, Xu P, Zhang D, Sun Z, et al. Deep-Learning Approach to Automatic Identification of Facial Anomalies in Endocrine Disorders. Neuroendocrinology (2020) 110(5):328-37. doi: 10.1159/ 000502211

19. Liu J, Dai Y, Lee Y, Yuan E, Wang Q, Wang L, et al. Pediatric Reference Intervals of Liver and Renal Function Tests From Birth to Adolescence in Chinese Children as Performed on the Olympus Au5400. Clin Chim Acta (2019) 490:142-6. doi: 10.1016/j.cca.2019.01.001

20. Li X, Wang D, Yang C, Zhou Q, Zhuoga SL, Wang LQ, et al. Establishment of Age- and Gender-Specific Pediatric Reference Intervals for Liver Function Tests in Healthy Han Children. World J Pediatr (2018) 14(2):151-9. doi: 10.1007/s12519-018-0126-x

21. Hicks MJ, Jones JF, Minnich LL, Weigle KA, Thies AC, Layton JM. AgeRelated Changes in T- and B-Lymphocyte Subpopulations in the Peripheral Blood. Arch Pathol Lab Med (1983) 107(10):518-23. 
22. Zierk J, Hirschmann J, Toddenroth D, Arzideh F, Haeckel R, Bertram A, et al. Next-Generation Reference Intervals for Pediatric Hematology. Clin Chem Lab Med (2019) 57(10):1595-607. doi: 10.1515/cclm-2018-1236

23. Lado-Abeal J, Rodriguez-Arnao J, Newell-Price JD, Perry LA, Grossman AB, Besser GM, et al. Menstrual Abnormalities in Women With Cushing's Disease are Correlated With Hypercortisolemia Rather Than Raised Circulating Androgen Levels. J Clin Endocrinol Metab (1998) 83(9):3083-8. doi: $10.1210 /$ jcem.83.9.5084

24. Bertagna X. Effects of Chronic ACTH Excess on Human Adrenal Cortex. Front Endocrinol (Lausanne) (2017) 8:43. doi: 10.3389/fendo.2017.00043

25. Pasternak-Pietrzak K, Moszczyńska E, Roszkowski M, Kot K, Marczak E, Grajkowska W, et al. Long-Term Outcome in Patients After Treatment for Cushing's Disease in Childhood. PloS One (2019) 14(12):e0226033. doi: 10.1371/journal.pone.0226033

26. Lonser RR, Wind JJ, Nieman LK, Weil RJ, DeVroom HL, Oldfield EH. Outcome of Surgical Treatment of 200 Children With Cushing's Disease. J Clin Endocrinol Metab (2013) 98(3):892-901. doi: 10.1210/ jc.2012-3604

27. Pivonello R, Isidori AM, De Martino MC, Newell-Price J, Biller BM, Colao A. Complications of Cushing's Syndrome: State of the Art. Lancet Diabetes Endocrinol (2016) 4(7):611-29. doi: 10.1016/s2213-8587(16) 00086-3

28. Fan L, Zhuang Y, Wang Y, Liu X, Liu D, Xiang B, et al. Association of Hypokalemia With Cortisol and ACTH Levels in Cushing's Disease. Ann N Y Acad Sci (2020) 1463(1):60-6. doi: 10.1111/nyas.14205

29. Tatsi C, Boden R, Sinaii N, Keil M, Lyssikatos C, Belyavskaya E, et al. Decreased Lymphocytes and Increased Risk for Infection are Common in Endogenous Pediatric Cushing Syndrome. Pediatr Res (2018) 83(2):431-7. doi: $10.1038 / \mathrm{pr} .2017 .278$
30. Kronfol Z, Starkman M, Schteingart DE, Singh V, Zhang Q, Hill E. Immune Regulation in Cushing's Syndrome: Relationship to Hypothalamic-PituitaryAdrenal Axis Hormones. Psychoneuroendocrinology (1996) 21(7):599-608. doi: 10.1016/s0306-4530(96)00021-2

31. Brunetti M, Martelli N, Colasante A, Piantelli M, Musiani P, Aiello FB. Spontaneous and Glucocorticoid-Induced Apoptosis in Human Mature T Lymphocytes. Blood (1995) 86(11):4199-205. doi: 10.1182/blood.V86. 11.4199.bloodjournal86114199

32. Pecori Giraldi F, Moro M, Cavagnini F. Gender-Related Differences in the Presentation and Course of Cushing's Disease. J Clin Endocrinol Metab (2003) 88(4):1554-8. doi: 10.1210/jc.2002-021518

Conflict of Interest: The authors declare that the research was conducted in the absence of any commercial or financial relationships that could be construed as a potential conflict of interest.

Publisher's Note: All claims expressed in this article are solely those of the authors and do not necessarily represent those of their affiliated organizations, or those of the publisher, the editors and the reviewers. Any product that may be evaluated in this article, or claim that may be made by its manufacturer, is not guaranteed or endorsed by the publisher.

Copyright (c) 2021 Zheng, Wang, Zhang, Feng, Liu, Li, Bao, Lu, Zhu, Feng and Wang. This is an open-access article distributed under the terms of the Creative Commons Attribution License (CC BY). The use, distribution or reproduction in other forums is permitted, provided the original author(s) and the copyright owner(s) are credited and that the original publication in this journal is cited, in accordance with accepted academic practice. No use, distribution or reproduction is permitted which does not comply with these terms. 